\title{
Apps para dejar de fumar mediante Terapia Cognitivo Conductual. Una revisión sistemática
}

\section{Apps for smoking cessation through Cognitive Behavioural Therapy. A review}

\author{
Patricia García-Pazo*,**, Joana Fornés-Vives*,**, Albert Sesé**,***, \\ Francisco Javier Pérez-Pareja***. \\ * Departamento de Enfermería y Fisioterapia. Universitat de les Illes Balears, Palma. España. \\ ** Institut d’Investigació Sanitària Illes Balears (IdISBa), Palma. España. \\ *** Departamento de Psicología. Universitat de les Illes Balears, Palma. España.
}

\section{Resumen}

El tabaquismo constituye un problema sanitario y económico de difícil erradicación. Las personas más dependientes a la nicotina suelen presentar, además, problemas psicopatológicos como depresión y ansiedad. Según las Guías de Práctica Clínica (GPC), el tratamiento recomendado para abandonar el hábito es la Terapia Cognitivo Conductual (TCC), sola o combinada con medicación. Actualmente, las aplicaciones móviles $(A p p)$ en salud $(m H e a l t h)$ permiten un acceso masivo y económico a este tratamiento. El objetivo de este trabajo consiste en llevar a cabo una revisión bibliográfica de las Apps para dejar de fumar que apliquen TCC y describir las técnicas implementadas. En el marco del protocolo PRISMA, la búsqueda se ciñó al periodo 2010-19 y se realizó en las bases de datos: EBSCOhost, Cochrane, Web of Science y Scopus. Se hallaron un total de 415 trabajos, de los cuales, tras aplicar los criterios de inclusión/exclusión, solo 5 artículos fueron objeto de revisión. Únicamente se identificaron 3 Apps (en inglés) que incluyeran TCC y, las técnicas más utilizadas fueron: el registro de cigarrillos, la visualización del progreso mediante gráficas, videos psicoeducativos, la motivación, el apoyo social mediante redes sociales y elementos de gamificación para reforzar la adherencia y la conducta de abstinencia. Los resultados sugieren incluir en este tipo de Apps el análisis de la conducta de fumar, ya que no todas lo hacen, así como una interfaz que comunique el personal sanitario con el usuario y pueda proporcionar un tratamiento personalizado.

Palabas clave: Dejar de fumar; Terapia Cognitivo Conductual; aplicaciones móviles.

\begin{abstract}
Smoking is a health and economic problem that is difficult to eradicate. Nicotine addicts also often suffer from psychological problems such as depression and anxiety. The recommended treatment according to clinical practice guidelines (CPG) is Cognitive Behavioural therapy (CBT), alone or combined with medication. Currently, health-related apps (m Health) enable wider availability and access to this treatment. The objectives of this study are to carry out a review of the smoking cessation apps that apply CBT and to describe the techniques used by them. Following the PRISMA framework, the databases EBSCOhost, Cochrane, Web of Science and Scopus were searched for publications between 2010-2019. Four hundred fifteen studies were found and, after applying the inclusion and exclusion criteria, only five articles were eligible for systematic review. Only three apps were identified as using $\mathrm{CBT}$, and the techniques most commonly used were the recording of smoking history, progress visualised through graphics, psycho-educational videos, motivation, social support through social media, and elements of gamification to reinforce adherence and abstinence behaviour. The results recommend the inclusion of smoking behaviour analysis in these types of apps, as not all of them do so, as well as an interface between the health professionals and the users to provide a personalised treatment.

Key Words: Smoking cessation; Cognitive Behavioural Therapy; mobile applications.
\end{abstract}




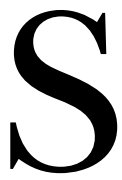

egún la Organización Mundial de la Salud (OMS), el tabaco ocasiona la muerte a la mitad de sus consumidores. Se estima que en el año 2030 habrá más de 7 millones de muertes por tabaco al año (OMS, 2019). En España, el porcentaje de fumadores diarios mayores de 15 años es superior al $22 \%$ de la población (ENSE, 2017). En población adolescente se ha encontrado un incremento del consumo entre los 17-18 años (Leal-López, Sánchez-Queija y Moreno, 2019). Además, el tabaco es una de las pocas drogas que no sólo perjudica al consumidor sino también a las personas de su alrededor.

Desde el punto de vista presupuestario, el tabaquismo impone una pesada carga económica en todo el mundo, especialmente en Europa y América del Norte, donde la epidemia de tabaco está más avanzada. En el Reino Unido, el sistema nacional de salud inglés (NHS), estimó que el coste sanitario anual derivado del tabaquismo fue de 2.600 millones de libras (UK Government, 2015). A nivel mundial, el importe por consumo de tabaco en 2012 ascendía al $5,7 \%$, sin contar los costes indirectos por pérdidas de productividad (Goodchild, Nargis y Tursan d'Espaignet, 2018). Tampoco se considera el gasto que supone el tabaquismo pasivo, responsable de alrededor de 600.000 muertes al año (Öberg, Jaakkola, Woodward, Peruga y Prüss-Ustün, 2011).

El consumo de tabaco, además de un grave problema sanitario y económico, es una adicción difícil de erradicar, por la dependencia que genera tanto a nivel fisiológico como psicológico y social. Su retirada provoca además, varias consecuencias: sintomatología de tipo consumo compulsivo pese al deseo e intentos de no fumar, efectos psicoactivos de la sustancia sobre el encéfalo y alteración del comportamiento por el reforzamiento de la nicotina (Camarelles et al., 2009). En relación con los efectos anteriores, cabe destacar que las personas con mayor dependencia a la nicotina presentan frecuentemente problemas psicopatológicos (ansiedad, depresión, estrés, etc.), hecho que hace que los tratamientos de deshabituación se tornen más complejos (Becoña et al., 2014). En el caso de la depresión se ha encontrado una relación lineal entre gravedad de consumo y gravedad de los síntomas de depresión (Jiménez-Treviño et al., 2019).

Los tratamientos incluidos en las Guías de Práctica Clínica (GPC) para la deshabituación al tabaco incluyen tratamientos farmacológicos (Terapia Sustitutiva de la Nicotina, Bupropion o Vareniclina), terapia de conducta de tipo Terapia Cognitivo Conductual (TCC) o la combinación de ambos en los casos más complejos (Fiore et al., 2008; NICE, 2018).

La TCC descrita en estos programas se centra fundamentalmente en el análisis de la conducta de fumar con el objetivo de desarrollar en el fumador conductas alternativas para afrontar de forma más eficaz situaciones de riesgo relacionadas con el hábito, lo cual ha demostrado ser eficaz para mantener la abstinencia (Deiches, Baker, Lanza y Piper, 2013). Los programas de TCC son descritos como terapias multicomponente ya que incorporan diferentes técnicas, como psicoeducación, reestructuración cognitiva, solución de problemas, relajación y apoyo social, entre otros, alrededor de una estrategia de abandono (Alonso-Pérez et al., 2014; Becoña, Míguez, Fernandez del Río y López, 2010; Raich et al., 2015). La necesidad de atender a un gran número de población y el gasto que genera este problema de salud ha obligado a que los tratamientos que han demostrado eficacia sean proporcionados de manera diferente al formato presencial, por ejemplo, a través del uso de tecnologías de la información y comunicación (TIC). Las TICS y, específicamente, las aplicaciones de salud de los teléfonos móviles, llamadas mHealth, pueden ofrecer una alternativa a un coste bajo. Además, estas Apps ofrecen a sus usuarios una serie de beneficios que no tienen otros formatos, tales como: accesibilidad a los tratamientos, atención de manera inmediata y en tiempo real a la conducta de fumar y a los síntomas de abstinencia, flexibilidad en el tiempo, seguimiento de los progresos, feedback personalizado, apoyo motivacional y complementariedad en cuanto a favorecer la comunicación con los profesionales de la salud (Kazdin, 2015; Kreps y Neuhauser, 2010; Do et al., 2018; Whittaker, McRobbie, Bullen, Rodgers y $\mathrm{Gu}, 2016)$. Un reflejo de estas ventajas es el crecimiento de dichas aplicaciones, en el mercado (Haskins, Lesperance, Gibbons y Boudreaux, 2017) y el número de descargas mensuales para dejar de fumar (Hartmann-Boyce, Stead, Cahill y Lancaster, 2013). No obstante, existen también algunos inconvenientes a tener en cuenta como: problemas técnicos (fallos informáticos de la aplicación), seguridad de los datos, privacidad del paciente, manejo del dispositivo por parte del profesional sanitario, o desconfianza del usuario en este tipo de método (Luxton, Mccann, Bush, Mishkind y Reger, 2011).

A pesar del elevado número de Apps y descargas, la comunidad científica informa que es necesario conocer la eficacia de los tratamientos que ofrecen, porque la gran mayoría de las mHealth, no se adaptan a las pautas que marcan las GPC (Abroms, Lee Westmaas, Bontemps-Jones, Ramani y Mellerson, 2013; Gulati y Hinds, 2018; Haskins et al., 2017; Thomas, Abramson, Bonevski y George, 2017) y tampoco incorporan terapias que han demostrado eficacia, como la TCC (Heather, Haffe, Peele y Rho, 2016; Nijhof, Bleijenberg, Uiterwaal, Kimpen y Putte, 2012).

En base a lo expuesto anteriormente nos planteamos si existen aplicaciones móviles para dejar de fumar que incluyan como tratamiento la TCC. Para responder a esta pregunta se formulan los siguientes objetivos: 1) Identificar si existen aplicaciones de telefonía móvil $(A p p)$ para dejar de fumar que incluyan TCC, y 2) Describir las técnicas de TCC que utilizan estas aplicaciones. 


\section{Método}

Se realizó una revisión bibliográfica utilizando las palabras clave: Smoking Cessation, mHealth, mobile application, Smartphone, Cognitive Behavioural Therapy, cbt, que fueron combinadas con los operadores booleanos clásicos (OR, AND) y búsqueda por frase utilizando (") y truncamiento (*). Se seleccionaron los artículos que contenían los descriptores en términos claves en el campo de título, abstract y palabras clave, en el periodo comprendido entre el año 2010 y agosto 2019. Se han consultado las bases de datos: EBscohost (PsycINFO, CINAHL, Psycarticles, Psychology and Behavioral Sciences Collection), Cochrane (PubMed, EMBASE), Web Of Science (Medline, Scielo) y Scopus. Todo el proceso de revisión ha seguido las recomendaciones del protocolo PRISMA (Liberati, Altman, Tetzlaff y Al, 2009).

Los criterios de inclusión fueron: 1) aparición de alguno de los términos de búsqueda en el título, resumen o palabra clave, 2) participantes de los estudios mayores de 18 años y 3) publicaciones en inglés o español. Se excluyeron los estudios realizados sobre muestras con patología mental, embarazadas y estudios que traten varias adicciones a la vez (ejemplo: alcohol y tabaco, marihuana y tabaco...).

Los registros encontrados se incorporaron al gestor de referencias Mendeley, y fueron eliminadas las duplicacio- nes. Mediante la lectura del título y del resumen se verificó que cumplieran con los criterios de inclusión/exclusión planteados, procediendo a descartar los excluidos. Posteriormente, se obtuvieron los textos completos de los seleccionados para ser evaluados en la totalidad de su extensión.

\section{Resultados}

La búsqueda permitió encontrar un total de 415 estudios: 16 en Scopus, 5 en EBSCOhost, 316 en Cochrane Library y 78 Web of Science. En la Figura 1 puede observarse el diagrama de flujo de la selección de artículos. Tras eliminar los trabajos que no cumplían con los criterios de inclusión quedaron un total de 6 estudios de los cuales se realizó un análisis exhaustivo. Finalmente, se eliminó otro estudio por no describir una App específica sino únicamente una técnica de TCC.

Uno de los principales aspectos observados en esta revisión es la existencia de un gran número de aplicaciones móviles que tienen como finalidad ayudar a dejar de fumar, puesto que se han identificado al menos 400 Apps disponibles, tanto para sistema operativo Android como iPhone (Regmi, Kassim, Ahmad y Tuah, 2017). Sin embargo, es escaso el número de mHealth encontradas que incorporen TCC. También, es importante señalar que, a pesar del gran

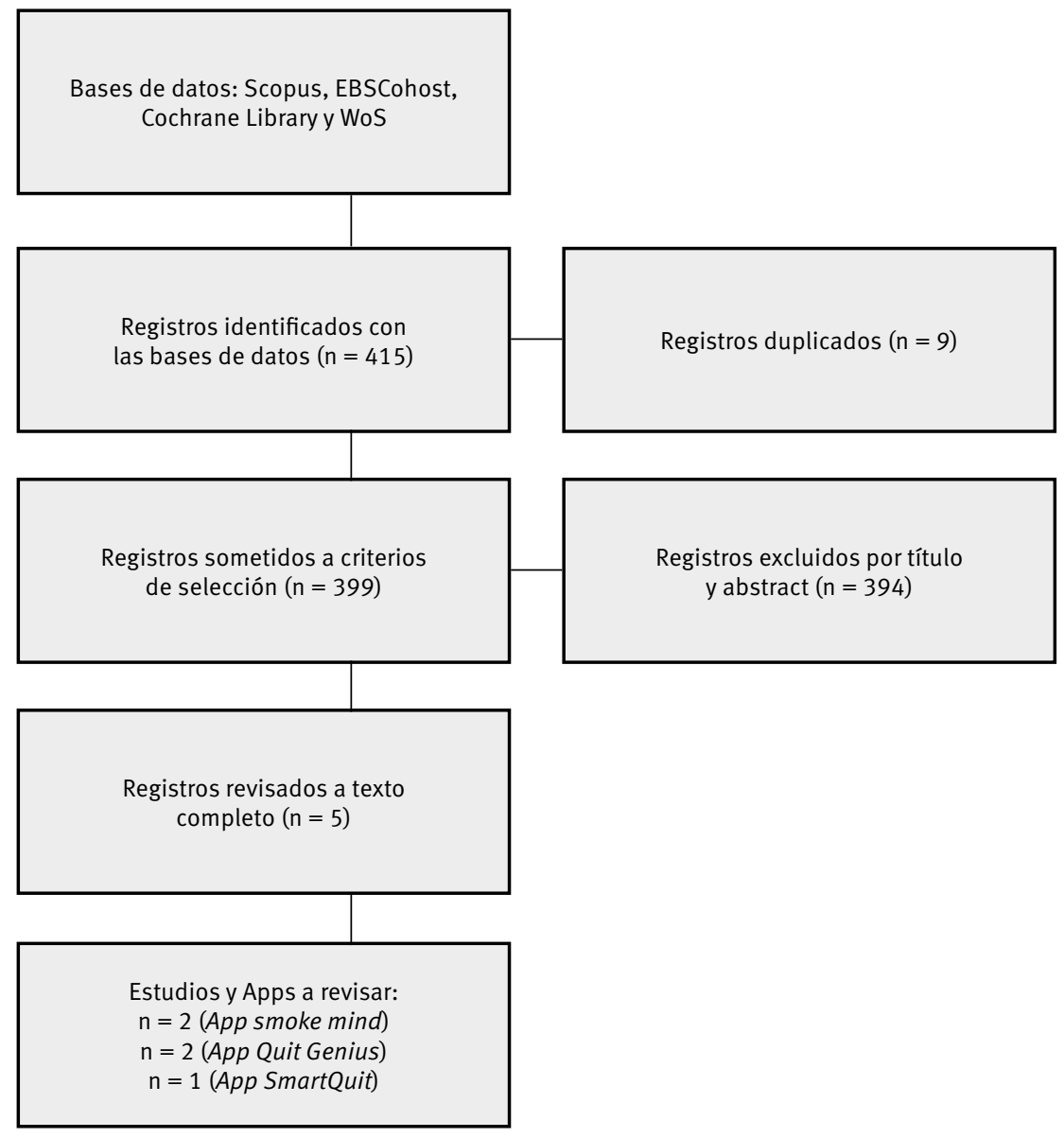

Figura 1. Diagrama de flujo de la selección de los estudios. 
número de aplicaciones disponibles (Apps store y Play store) para tratar el tabaquismo, no hay estudios que hayan probado su eficacia ni están respaldadas por personal sanitario (Haskins et al., 2017).

De los 5 artículos seleccionados, se extraen los nombres de 3 Apps para dejar de fumar que incorporan TCC: SmartQuit (Heffner, Vilardaga, Mercer, Kientz y Bricker, 2015), Smoke Mind (Alsharif y Philip, 2015a, 2015b) y Quit Genius (Lin et al., 2018; Tudor-Sfetea et al., 2018). Tras un análisis exhaustivo de los estudios seleccionados se ha intentado extraer de cada uno aquella información útil y en consonancia con los objetivos de nuestro trabajo (Tabla 1).

El primer estudio presenta la App SmartQuit (Heffner et al., 2015), diseñada en Seattle por uno de los coautores el Dr. Bricker (Fred Hutchinson Cancer Research Center). Se limita a los usuarios de habla inglesa con dispositivos de tipo Apple. Presenta un tratamiento de 8 semanas basado en Terapia de Aceptación y Compromiso (ACT), combi- nado con técnicas de TCC. A los 60 días tras el uso de la $A p p$, evalúan sobre un total de 41 funciones disponibles, las 10 más utilizadas. Sus resultados indican que 8 son de TCC (ver tabla 1), hallando una relación estadísticamente significativa entre la función "consultar plan de abandono" y dejar de fumar. Esta función propone actividades como: fijar una fecha de cese del hábito, indicar los motivos por los que desea abandonar el tabaco (apoyados mediante imágenes), consensuar la estrategia comportamental para conseguir reducir el consumo e indicar si se apoyaran con medicación. También los usuarios pueden consultar sus hábitos de consumo, su gasto económico mensual y seleccionar a las personas de su círculo que le apoyaran durante el proceso de abandono.

Los dos estudios que hacen referencia a la App, Smoke Mind están publicados en el año 2015 por los mismos autores, Alsharif y Philip. El primer estudio presenta una propuesta de diseño de una aplicación sobre un modelo

Tabla 1. Resultados de los artículos revisados.

\begin{tabular}{|c|c|c|c|c|c|}
\hline $\begin{array}{l}\text { Autor } \\
\text { (Año) }\end{array}$ & Objetivos & $\begin{array}{c}\text { Diseño/Muestra/ } \\
\text { Medidas }\end{array}$ & $\begin{array}{l}\text { Nombre App/ } \\
\text { Características }\end{array}$ & Componentes TCC & Resultados \\
\hline $\begin{array}{l}\text { Heffner } \\
\text { et al. } \\
(2015)\end{array}$ & $\begin{array}{l}\text { (1) Especificar las } 10 \\
\text { funciones más utiliza- } \\
\text { das de la aplicación } \\
\text { para dejar de fumar. } \\
\text { (2) Determinar cuáles } \\
\text { son predictores del } \\
\text { abandono de tabaco. }\end{array}$ & $\begin{array}{l}\text { Análisis descriptivo } \\
\text { (post hoc). } \\
\qquad \mathrm{N}=76 \\
\text { Género }=54 \%(\mathrm{H}) \\
\text { Edad }(\mathrm{m} / \mathrm{dt})= \\
(41,8 / 11,9) \\
\text { Cuestionario online } \\
\text { sobre aspectos } A P P \\
\text { online. }\end{array}$ & $\begin{array}{c}\text { App: Smart Quit } \\
\text { Características: } \\
\text { Combina ACT y TCC. }\end{array}$ & $\begin{array}{l}\text { - Autoregistros de la conducta } \\
\text { con feedback (seguimientos y } \\
\text { progresos). } \\
\text { - Fijación de metas (plan de } \\
\text { abandono). } \\
\text { - Reforzamiento positivo (ele- } \\
\text { mentos de gamificación). } \\
\text { - Apoyo social (compartir pro- } \\
\text { gresos). }\end{array}$ & $\begin{array}{l}\text { (1) Sólo } 8 \text { de las } 10 \text { funciones, } \\
\text { son TCC; ver el plan de abando- } \\
\text { no, seguimientos de la conduc- } \\
\text { ta de fumar, ver progresos en } \\
\text { calendario, ver página de com- } \\
\text { partir, ver progresos en la tabla, } \\
\text { ver seguimientos de los lugares, } \\
\text { ver insignias ganadas y block de } \\
\text { notas. } \\
\text { (2) Mejor predictor del cese de } \\
\text { fumar, ver el plan de abandono } \\
(p=0,03) \text {. OR }=11,1,95 \% \mathrm{Cl}= \\
1,3-94,2 \text {. Predictor de fracaso, } \\
\text { ver seguimientos } p<0,04, \mathrm{OR}= \\
0,11,95 \% \mathrm{Cl}(0,1-0,9) .\end{array}$ \\
\hline $\begin{array}{l}\text { Alsharif } \\
\text { y Philip } \\
(2015)\end{array}$ & $\begin{array}{l}\text { Proponer un mode- } \\
\text { lo que incluya TCC y } \\
\text { tecnología móvil para } \\
\text { dejar de fumar. }\end{array}$ & $\begin{array}{l}\text { Exposición de conte- } \\
\text { nidos sobre los obje- } \\
\text { tivos planteados. }\end{array}$ & $\begin{array}{c}\text { App: Smoke Mind } \\
\text { Características: } \\
\text { Portal web para los profe- } \\
\text { sionales sanitarios. } \\
\text { Monitorización remota } \\
\text { Canal comunicativo con } \\
\text { terapeuta TCC. }\end{array}$ & $\begin{array}{l}\text { Feedback directo y personal } \\
\text { con profesionales de la salud. } \\
\text { - Gráficos de la conducta. } \\
\text { - Apoyo emocional. } \\
\text { - Análisis de la conducta (pien- } \\
\text { so, siento y actúo). } \\
\text { - Psicoeducación (videos). } \\
\text { - Entrenar habilidades. } \\
\text { - Relajación. } \\
\text { - Solución de problemas. } \\
\text { - Mensajes motivacionales. } \\
\text { - Apoyo social. }\end{array}$ & $\begin{array}{l}\text { El modelo propuesto consta de: } \\
\text { - Plataforma mHealth. } \\
\text { - Sistema de soporte basado en } \\
\text { técnicas de minería de datos } \\
\text { para proveer de un manejo de } \\
\text { la información óptimo. }\end{array}$ \\
\hline $\begin{array}{l}\text { Alsharif } \\
\text { y Philip } \\
\text { (2015) }\end{array}$ & $\begin{array}{l}\text { Conocer las opiniones } \\
\text { respecto a las prefe- } \\
\text { rencias de fumadores } \\
\text { y profesionales de sa- } \\
\text { lud, en el uso de la App } \\
\text { para dejar de fumary } \\
\text { las funciones de esta. }\end{array}$ & $\begin{array}{l}\text { Estudio descriptivo. } \\
\qquad \begin{array}{c}\mathrm{N}=52 \\
\text { Género }=71 \%(\mathrm{H}) \\
\text { Mujeres }=29 \% \\
\text { Edad }=18-54 \\
\text { Cuestionario basado } \\
\text { en conceptos de uso } \\
\text { de la App y entrevista } \\
\text { semiestructurada. }\end{array}\end{array}$ & $\begin{array}{l}\text { App: Smoke Mind } \\
\text { (mismas características } \\
\text { estudio anterior) }\end{array}$ & $\begin{array}{l}\text { (mismas características del es- } \\
\text { tudio anterior) }\end{array}$ & $\begin{array}{l}\text { Preferencias de usuarios: } \\
\text { - Gráficas de progreso ( } 90 \%) \text {. } \\
\text { - Alertas; notificaciones }(57,6 \%) \text {. } \\
\text { - Videos informativos, material } \\
\text { educativo, feedback de médi- } \\
\text { cos y de exfumadores }(88,4 \%) \text {. } \\
\text { - Teléfono de ayuda ( } 77 \%) \text {. } \\
\text { - Seguir con la app al finalizar } \\
\text { tratamiento (73\%). } \\
\text { Preferencias de los profesionales: } \\
\text { - Interacciona con el hospital. } \\
\text { - Poder actualizar los seguimien- } \\
\text { tos. } \\
\text { - Poder atender a los problemas } \\
\text { de forma individual. }\end{array}$ \\
\hline
\end{tabular}


de tratamiento que combina TCC con mHealth (Alsharif y Philip, 2015a). La App dispone de 3 módulos: módulo de manejo del cese para dejar de fumar, modulo interactivo (dispone de una interfaz donde los profesionales pueden intervenir en el tratamiento) y módulo de TCG. Las funciones de TCC se centran en identificar las situaciones de riesgo (situaciones en las que fumaba y ahora debe mantenerse abstinente) y en trabajar sobre ellas con el objetivo de modificar la conducta. Para cumplir este objetivo, incorporan técnicas como: reestructuración cognitiva, solución de problemas, entrenamiento en habilidades de afrontamiento y relajación entre otras (ver Tabla 1). Estas

Tabla 1 (cont.). Resultados de los artículos revisados.

\begin{tabular}{|c|c|c|c|c|c|}
\hline $\begin{array}{l}\text { Autor } \\
\text { (Año) }\end{array}$ & Objetivos & $\begin{array}{c}\text { Diseño/Muestra/ } \\
\text { Medidas }\end{array}$ & $\begin{array}{l}\text { Nombre App/ } \\
\text { Características }\end{array}$ & Componentes TCC & Resultados \\
\hline $\begin{array}{l}\text { Tudor- } \\
\text { Sfetea et } \\
\text { al. (2018) }\end{array}$ & $\begin{array}{l}\text { (1) Identificar las opi- } \\
\text { niones sobre la App } \\
\text { como método para de- } \\
\text { jar de fumar y respecto } \\
\text { a sus funciones respec- } \\
\text { to } 2 \text { apps (Smokefree } \\
\text { (No TCC) y Quit Genius } \\
\text { (SI TCC)). } \\
\text { (2) Identificar las opi- } \\
\text { niones de los usuarios } \\
\text { de las apps respecto a } \\
\text { los resultados. }\end{array}$ & 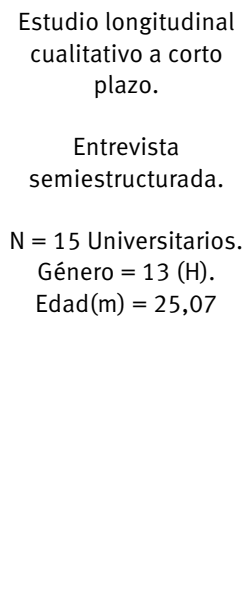 & 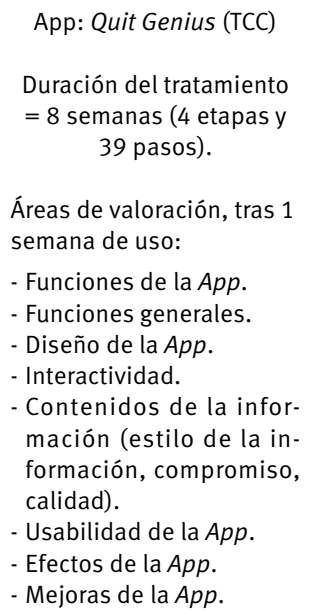 & $\begin{array}{l}\text { - Registro de la conducta (regis- } \\
\text { trar cigarrillos). } \\
\text { - Mensajes motivacionales. } \\
\text { - Información educativa (sobre } \\
\text { consecuencias del tabaco). } \\
\text { - Psicoeducación (sobre la } \\
\text { TCC). } \\
\text { - Relación personalizada. } \\
\text { - Ayuda a identificar detonado- } \\
\text { res de la conducta de fumar. } \\
\text { - Exposición en imaginación } \\
\text { (Situaciones críticas). } \\
\text { - Relajación. } \\
\text { - Solución de problemas. } \\
\text { - Elementos de gamificación } \\
\text { - Apoyo social (comunidad en- } \\
\text { tre iguales). }\end{array}$ & $\begin{array}{l}\text { (1) Los usuarios informan positi- } \\
\text { vamente respecto a la APP como } \\
\text { método y frente a las funciones, } \\
\text { como también en el diseño. } \\
\text { (2) Respecto a las opiniones de } \\
\text { sus resultados: } \\
\text { - Disminuye el número de ciga- } \\
\text { rrillo día (53\%). } \\
\text { - Expresan ha aumentado su } \\
\text { motivación para dejar de fumar } \\
\text { (53\%). } \\
\text { - Expresan su deseo de conti- } \\
\text { nuar usando la APP (67\%). } \\
\text { - Recomendarían la App a otra } \\
\text { persona (73\%). }\end{array}$ \\
\hline
\end{tabular}

\begin{tabular}{|c|c|c|c|c|c|}
\hline $\begin{array}{l}\text { Lin et al. } \\
(2018)\end{array}$ & $\begin{array}{l}\text { (1) Explorar la función } \\
\text { del progreso del pro- } \\
\text { grama de la TCC. } \\
\text { (2) Examinar los ele- } \\
\text { mentos de gamificación } \\
\text { del diseño de la App, } \\
\text { sobre los constructos: } \\
\text { empoderamiento, bien- } \\
\text { estar e inspiración. } \\
\text { (3) Explorar la rela- } \\
\text { ción de los construc- } \\
\text { tos:empoderamiento, } \\
\text { bienestar e inspiración } \\
\text { sobre dejar de fumar o } \\
\text { reducir el número de } \\
\text { cigarrillos fumados. } \\
\text { (4) Identificar y des- } \\
\text { cribir los posibles fa- } \\
\text { cilitadores y barreras } \\
\text { que surgen de los ele- } \\
\text { mentos de diseño de } \\
\text { la aplicación. }\end{array}$ & $\begin{array}{l}\text { Estudio longitudinal } \\
\text { cualitativo. } \\
\qquad \mathrm{N}=190 \\
\text { Edad }(\mathrm{m})=36 \\
\text { Género }=52,6 \%(\mathrm{M}) \\
\text { Entrevista online. } \\
\text { Escalas a daptadas } \\
\text { para medir ítems: } \\
\text { Bienestar hedónico, } \\
\text { empoderamiento, ins- } \\
\text { piración y ansiedad. }\end{array}$ & $\begin{array}{l}\text { App: Quit Genius } \\
\text { (mismas características } \\
\text { estudio anterior) }\end{array}$ & $\begin{array}{l}\text { (mismas características del es- } \\
\text { tudio anterior) }\end{array}$ & 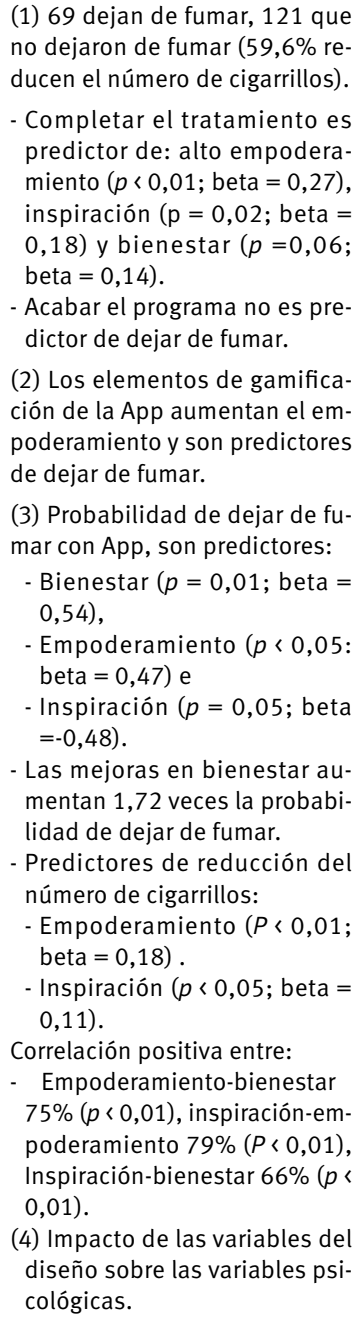 \\
\hline
\end{tabular}


técnicas se explican mediante videos educativos, tutoriales y/o mensajes. Además, Smoke Mind dispone de un registro diario del monóxido de carbono (CO) del usuario, realizado mediante un cooxímetro que dispone el paciente. Una vez realizada la medida, la transfiere manualmente a la $A p p$ y ésta envía la información al centro. Ante un aumento de $\mathrm{CO}$, el terapeuta en TCC, solicita al usuario un análisis de la conducta (pienso, siento y actúo) y da un feedback en el momento.

El segundo trabajo sobre la aplicación Smoke Mind (Alsharif y Philip, 2015b), tiene como objetivo valorar las opiniones que tienen sus usuarios y profesionales de salud sobre la $A p p$ como método para dejar de fumar, así como de sus funciones. Para ello, se entrevista a una muestra de 52 usuarios, en su mayoría universitarios y personal sanitario (3 doctores y 5 trabajadores sociales). Los resultados indican que el $100 \%$ de los usuarios valoran positivamente recibir el tratamiento para dejar de fumar mediante la $A p p$, resaltando algunas preferencias (ver Tabla 1). Por otra parte, el personal sanitario valora como algo importante poder intervenir en el tratamiento e interactuar con el paciente para abordar los problemas de forma individual.

Los dos últimos registros revisados analizan Quit Genius, (Lin et al., 2018; Tudor-Sfetea et al., 2018) que presenta un tratamiento de TCC distribuido en 4 etapas y dividido en 39 pasos. Además, propone una aplicación personalizada que implica la autorreflexión de la conducta de fumar, el registro de consumo de cigarrillos, la psicoeducación (formato video y audio), el desarrollo de estrategias de afrontamiento, ejercicios interactivos (motivación, relajación, distracción y gestión del estrés) y técnicas de solución de problemas. Dispone también de elementos de gamificación para reforzar la conducta de abstinencia del tabaco bajo un supuesto viaje con logros, barras de progreso, seguimiento, insignias de los logros y una calculadora que muestra el ahorro monetario al disminuir el consumo. El primer estudio de Lin et al. (2018) se plantea el objetivo de explorar en la $A p p$ el papel que tiene el tratamiento TCC y los elementos de gamificación sobre componentes psicológicos como empoderamiento (motivación intrínseca), bienestar e inspiración, entre otros. Además, evalúan la efectividad de estos elementos sobre el abandono del tabaco o reducción del consumo de cigarrillos. Los datos a estudio son recogidos por la misma aplicación y por una entrevista online realizada al usuario justo al finalizar el tratamiento. Sus resultados indican que realizar el tratamiento de TCC completo es predictor de aumento de los constructos: bienestar, inspiración y empoderamiento. Mediante un análisis de regresión identifican bienestar como predictor de abandono del tabaco, mientras que el aumento de la inspiración es predictor de mantenerse como fumador, pero con un 34\% de probabilidad de reducción del número de cigarrillos por día. El empoderamiento se relaciona también con una disminución del número de ci- garrillos. Resaltan el impacto de los elementos del diseño de la $A p p$ sobre el aumento de estas variables, igualmente, se videncia que los elementos de gamificación aumentan el empoderamiento del usuario, prediciendo la capacidad de abandono.

En el estudio de Tudor-Sfetea et al. (2018) se evalúa las opiniones de los usuarios respecto a la $A p p$, valorando tanto la aplicación como método para ayudar a dejar de fumar, como sus funciones y su posible influencia en los cambios de comportamiento. Los datos se obtienen a través de entrevistas realizadas a 15 universitarios, los cuales dieron una valoración positiva tanto sobre sus funciones como en el uso de la App como método para dejar de fumar. En los resultados de este estudio se valora de forma positiva el diario de la conducta de fumar, entendida como una técnica que aumenta la motivación para el abandono del hábito y para disminuir el consumo de cigarrillos. También fueron valorados de forma muy positiva los elementos de gamificación.

Cada uno de los cinco trabajos revisados exponen las técnicas TCC que implementan las Apps, pero solamente uno de ellos describe el desarrollo de la aplicación y su funcionamiento (Alsharif y Philip, 2015a). En la Tabla 2 puede observarse un resumen de las técnicas fundamentales en las intervenciones de un tratamiento con TCC y aquellas que los estudios sobre las Apps revisadas describen. Las principales técnicas TCC implementadas por las 3 aplicaciones son: el registro de cigarrillos, la visualización del progreso mediante gráficas, la psicoeducación (mediante videos educativos, tutoriales y/o textos), la motivación (mediante videos, mensajes motivacionales), el apoyo social (mediante el uso de redes sociales), ofrecer la posibilidad de un tratamiento combinado con medicación, y también, añadir elementos de gamificación para reforzar la conducta de abstinencia y/o utilización de la $A p p$.

\section{Discusión}

El objetivo principal de este estudio era determinar la existencia de posibles aplicaciones móviles para dejar de fumar y describir sus principales funciones, especialmente aquellas que contienen técnicas y/o procedimientos implícitos en TCC. Se han encontrado 3 aplicaciones: SmartQuit, Smoke Mind y Quit Genius, sin embargo, ninguno de los trabajos describe con amplitud las técnicas e información que incluyen las Apps. Para solventar este vacío de información, hemos intentado descargar las aplicaciones, consultar las páginas webs e incluso contactar con los autores de los estudios revisados. Aun así, ha sido difícil resolver algunas de las dudas sobre funcionalidad e información que ofrecen las aplicaciones.

Por otra parte, los estudios revisados hacen referencia a muestras pequeñas y no representativas de la población general, dificultando con ello la extrapolación de los resulta- 
Tabla 2. Componentes de la TCC que tienen las aplicaciones de móvil revisadas.

\begin{tabular}{|c|c|c|c|}
\hline TCC & SmartQuit & $\begin{array}{l}\text { Smoke } \\
\text { Mind }\end{array}$ & $\begin{array}{l}\text { Quit } \\
\text { Genius }\end{array}$ \\
\hline Evaluación mediante cuestionario: Dependencia a la nicotina & & $x$ & \\
\hline Entrevista sobre: Historia de tabaco (hábitos) & $\mathrm{x}$ & $x$ & $x$ \\
\hline Psicoeducación sobre conducta adictiva y TCC & & $x$ & $X^{*}$ \\
\hline Autoregistro del número de cigarrillos fumados & $\mathrm{x}$ & $x$ & $x$ \\
\hline Análisis de la conducta de fumar: (Pienso, siento y hago) & & $x$ & $x$ \\
\hline Plan de abandono: Técnicas de Reducción. Reducción gradual de la ingestión de nicotina y alquitrán & $x$ & $x$ & $x$ \\
\hline \multicolumn{4}{|l|}{ Plan de abandono: Fecha de abandono (eliminar todos los cigarrillos en esa fecha) } \\
\hline Ejercicios de motivación: Ventajas y desventajas de dejar de fumar. Razones-Motivos para dejar de fumar & $\mathrm{x}$ & $X^{*}$ & $x$ \\
\hline Control de la ansiedad, estrés: Entrenamiento en técnicas de relajación y respiraciones profundas & & $x$ & $x$ \\
\hline Control estímulos: Lugares, personas y actividades donde fumaba y ahora se mantendrá abstinente & & $x$ & $x$ \\
\hline Apoyo social: Ejercicio de compromiso social con personas del entorno o iguales & $x$ & $x$ & $x$ \\
\hline $\begin{array}{l}\text { Estrategias de afrontamiento generar conductas alternativas para mantener la abstinencia y } \\
\text { para enfrentarse a los síntomas del síndrome de la abstinencia de la nicotina }\end{array}$ & & $\mathrm{x}$ & $x$ \\
\hline Técnica de solución de problemas & & $x$ & \\
\hline Reestructuración cognitiva: Información sobre la identificación y el cambio de pensamiento & & $x$ & $x$ \\
\hline \multicolumn{4}{|l|}{ Autoinstrucciones } \\
\hline \multicolumn{4}{|l|}{ Ensayo conductual: Exponerse a situaciones en las que fumaba sin fumar } \\
\hline \multicolumn{4}{|l|}{ Desarrollo técnicas de rechazo de cigarrillos: Técnica de banco de niebla, disco rayado } \\
\hline Cooximetría (control de CO en el aire expirado) & & $x$ & $x$ \\
\hline Considera tratamiento combinado con medicación & $\mathrm{x}$ & $x$ & $x$ \\
\hline
\end{tabular}

Nota. TCC: Terapia Cognitivo-Conductual, CO: Monóxido de Carbono. $X^{\star}$ : No se especifica claramente el tipo de información.

dos. En el trabajo de Tudor-Sfetea et al. (2018) la muestra está constituida por 15 universitarios y en el de Alsharif y Philip (2015b) por 52 fumadores, en su mayoría también universitarios. Ambos estudios se realizan a través de diseños de tipo descriptivo y observacional, mientras que las dos investigaciones con la App Quit Genius realizan estudios longitudinales de tipo cualitativo. Este tipo de diseños no permiten obtener una relación causal entre las variables estudiadas en el tratamiento y los resultados obtenidos (dejar de fumar o reducir el número de cigarrillos). Igualmente, respecto al rigor científico de las fuentes publicadas, señalar que los estudios realizados con la App Quit Genius son revistas de alto impacto científico, sin embargo, no ocurre lo mismo con los estudios de las otras dos Apps, incluso uno de ellos (el de Smoke Mind), ha sido publicado en Proceedings de congresos.

Aunque las tres Apps aplican razonablemente una TCC, SmartQuit y Quit Genius la combinan con otra aproximación terapéutica como la ACT, considerada una Terapia de Tercera Generación y con técnicas de Mindfulness, respectivamente. Los estudios que en formato presencial han incorporado otras técnicas a los programas TCC, como por ejemplo la activación conductual, han aportado evidencia de mejora de los síntomas depresivos relacionados con las recaídas en el tabaquismo (Martínez-Vispo et al., 2019).
Sin embargo, no se dispone de evidencia que permita evaluar el efecto combinado de estas terapias o técnicas en las Apps revisadas. Por otra parte, el análisis de la conducta de fumar (pienso, siento y actúo) como técnica imprescindible en la TCC, aparece bien representada en las Apps Smoke Mind y Quit Genius, pero no puede afirmarse con rotundidad que se realice en el caso de SmartQuit. En esta última, cuyo objetivo es desarrollar habilidades para aceptar los desencadenantes de la conducta de fumar, tiene más peso la aproximación de las ACT.

Conscientes de que un tratamiento TCC debe incluir la sensibilización e información del fumador en el análisis de la conducta de las situaciones en las que fuma o fumaba, entendidas como situaciones críticas para las recaídas, consideramos que las $m$ Health pueden ayudar a identificarlas y registrarlas. Incluso es recomendable que las mismas aplicaciones notifiquen esta función para que el fumador esté atento a su progreso (Naughton, 2016). Desde esta perspectiva, consideramos que Smoke Mind proporciona al usuario mayor control sobre este análisis. Mediante una interfaz que dispone el personal sanitario, un terapeuta TCC puede ayudar al fumador a realizar el análisis en el momento, recibiendo así una atención personalizada. De ese modo, la interfaz puede facilitar la TCC vía $A p p$, además, permitir la interacción con el personal sanitario de refe- 
rencia, realizar mejores seguimientos y facilitar el acceso al sistema sanitario de los fumadores que quieren dejar de fumar.

Los contactos con el personal sanitario en las terapias a distancia como "Quitlines" han demostrado eficacia, pero según algunos autores (Stead, Koilpillai y Lancaster, 2015), presentan el inconveniente de ser infrautilizados por los fumadores. Este problema, coincide con los resultados del estudio de Alsharif y Philip (2015b), en el cual informan de que a pesar de existir la posibilidad de contactar con los profesionales de salud a través de la aplicación del móvil, más del $70 \%$ de la muestra nunca contactó. El estudio sobre SmartQuit (Heffner et al., 2015) también refiere problemas de infrautilización de las funciones disponibles en la $A p p$. Con el fin de solucionar los problemas anteriores y conseguir una respuesta más activa del usuario, es recomendable incorporar notificaciones o avisos del propio dispositivo (Naughton, 2016) e incluir elementos de gamificación en las mHealth (Edwards et al., 2016), pues mediante el juego, se puede reforzar la adherencia al tratamiento y el seguimiento tras la abstinencia (Andújar-Espinosa, Salinero-González, Castilla-Martínez, Castillo-Quintanilla, Ibañez-Meléndez y Hu-Yang, 2018). Ambas estrategias han sido incluidas por las aplicaciones revisadas y han sido valoradas positivamente por sus usuarios (Lin et al., 2018; Tudor-Sfetea et al., 2018).

A pesar de la relevancia científica y aplicada que tiene medir la eficacia del tratamiento tabáquico mediante valoraciones objetivables como reducir los síntomas de depresión, los síntomas de ansiedad, el craving, la disminución del número de cigarrillos y, por supuesto, dejar de fumar, la mayoría de estos estudios utilizan entrevistas semiestructuradas sobre las opiniones del uso de la $A p p$, ya sea por parte de los usuarios o el personal sanitario. Así, los estudios establecen valoraciones a partir de a) las opiniones de usuarios y profesionales (Alsharif y Philip, 2015a, 2015b), b) las funciones de la aplicación más utilizadas como predictoras de cese en el estudio de Heffner et al. (2015), o c) la predicción de dejar de fumar por la potenciación de constructos psicológicos (empoderamiento, bienestar e inspiración) (Lin et al., 2018). Con el fin de mejorar la calidad de la evidencia resultarían necesarias las evaluaciones antes y después del uso de la aplicación, la incorporación de pruebas objetivas (cooximetría, nicotina en saliva...) y/o información que puedan aportar personas allegadas al usuario o del personal sanitario, puesto que ningún estudio revisado incluye estas fuentes de objetivación y triangulación.

Otra limitación importante detectada en los estudios revisados está relacionada con el hecho de que las opiniones registradas son valoradas muy a corto plazo tras el uso de la App: sin haber finalizado el tratamiento (Alsharif y Philip, 2015b; Heffner et al., 2015; Tudor-Sfetea et al. 2018), o justo al acabar el tratamiento (Lin et al., 2018). Hay evidencia científica de que los porcentajes de recaídas de los fumadores son altos, desde el primer mes tras la abstinencia y van disminuyendo a los 3 meses, a los 6 meses y a los 12 meses de seguimiento en tratamientos para dejar de fumar (Minami et al., 2018). Ante esta carencia se debería llevar a cabo seguimientos con valoraciones objetivas más a largo plazo en los fumadores que mantienen la abstinencia tras el uso de la aplicación.

A modo de conclusión, la revisión ha puesto de relieve la existencia de un número reducido de aplicaciones para dejar de fumar que incluyan la TCC. Las Apps identificadas presentan limitaciones importantes: ofrecen poco nivel de detalle en la información respecto a las técnicas que realizan en su programa TCC, aunque explican algunos de los procedimientos que emplean, deberían detallar cómo lo hacen, la secuencia y la frecuencia con que los utilizan, de esta forma facilitaría la estandarización del programa. Además, no focalizan el trabajo sobre el análisis de la conducta de fumar, y en general no hacen referencia a niveles conseguidos de abstinencia al no incluir procedimientos suficientemente eficientes para evaluar la eficacia de la herramienta. Por todo ello, y de cara a la construcción de nuevas Apps para dejar de fumar, se recomienda la utilización de notificaciones y avisos, además de elementos de gamificación que refuercen la realización de las actividades propuestas, para que con ello traten de aumentar la adherencia al tratamiento y el consiguiente cambio de conducta. Para las funciones que precisan de mayor interacción, como el análisis de conducta, se recomienda incluir una interfaz que pueda facilitarlo, además de poder personalizar los tratamientos. De esta forma la $A p p$ se convertiría en un instrumento de soporte para el fumador. La inclusión de procedimientos de medición objetiva como el uso de cooxímetro y/o de información de la red social del usuario resulta necesario para aumentar la fiabilidad, tanto del proceso como del resultado de la aplicación de la herramienta. Por último, se recomienda desarrollar aplicaciones que permitan al usuario escoger su idioma para participar en la mHealth, y que, por su simplicidad, presenten un procedimiento de uso suficientemente intuitivo y sencillo como para que ni el nivel cultural ni las habilidades tecnológicas generen una brecha de uso.

\section{Reconocimientos}

Este estudio ha sido financiado por el Colegio Oficial de Enfermería de las Islas Baleares en el marco de las Ayudas a Proyectos de Investigación (PI-2019/0254).

\section{Conflicto de intereses}

Los autores declaran que no existe conflicto de intereses en ningún aspecto de esta investigación. 


\section{Referencias}

Abroms, L. C., Lee Westmaas, J., Bontemps-Jones, J., Ramani, R. y Mellerson, J. (2013). A content analysis of popular smartphone apps for smoking cessation. American Journal of Preventive Medicine, 45, 732-736. doi:10.1016/j. amepre.2013.07.008.

Alonso-Pérez, F., Alonso-Cardeñoso, C., García-González, J. V., Fraile-Cobos, J. M., Lobo-Llorente, N. y Secades-Villa, R. (2014). Efectividad de un programa multicomponente para dejar de fumar aplicado en atención primaria. Gaceta Sanitaria, 28, 222-224. doi:10.1016/j. gaceta.2013.11.002.

Alsharif, A. H. y Philip, N. (2015a). Cognitive behavioural therapy embedding smoking cessation program using smartphone Technologies. En: Libro de actas 5th World Congress on Information and Communication Technologies (WICT 2015) (pp. 134-139), Marrakech. doi:10.1109/ WICT.2015.7489660.

Alsharif, A. H. y Philip, N. Y. (2015b). A framework for smoking cessation in the Kingdom Of Saudi Arabia using smart mobile phone technologies (Smoke Mind). En: Libro de Actas 2nd International Conference on Computer Science, Computer Engineering, and Social Media (CSCESM 2015) (pp. 96-102), Polonia. doi:10.1109/CSCESM.2015.7331875.

Andújar-Espinosa, R., Salinero-González, L., Castilla-Martínez, M., Castillo-Quintanilla, C., Ibañez-Meléndez, R. y Hu-Yang, C. (2018). Evaluación de aplicaciones para la deshabituación tabáquica con elementos de gamificación: elaboración y aplicación de un check-list. Revista Española de Comunicación en Salud, 9, 152-162. doi:10.20318/recs.2018.4493.

Becoña, E., Fernández, E., López-Durán, A., Martínez, Ú., Martínez, C. y Rodriguez, R. A. (2014). El tratamiento psicológico de la dependencia del tabaco. Eficacia, barreras y retos para el futuro. Papeles Del Psicólogo, 35, 161-168.

Becoña, E., Míguez, M. C., Fernandez del Río, E. y López, A. (2010). El tratamiento psicológico de los fumadores. En E. Becoña (Ed.), Dependencia del tabaco. Manual de casos clínicos (pp. 43-60). Madrid, España: Sociedad Española de Psicología Clínica, Legal y Forense.

Camarelles, F., Salvador, T., Ramón, J. M., Córdoba, R., Jiménez, C., López, V.,... López, A. (2009). Consenso sobre la atención sanitaria del tabaquismo en España. $R$ vista Española de Salud Pública, 83, 175-200. doi:10.1590/ S1135-57272009000200004.

Deiches, J. F., Baker, T. B., Lanza, S. y Piper, M. E. (2013). Early lapses in a cessation attempt: Lapse contexts, cessation success, and predictors of early lapse. Nicotine $\mathcal{E}$ Tobacco Research : Official Journal of the Society for Research on Nicotine and Tobacco, 15, 1883-1891. doi:10.1093/ntr/ntt074.

Do, H. P., Tran, B. X., Le Pham, Q., Nguyen, L. H., Tran, T. T., Latkin, C. A.,... Baker, P. R. (2018). Which eHealth interventions are most effective for smoking cessation?
A systematic review. Patient Preference and Adherence, 12, 2065-2084. doi:10.2147/PPA.S169397.

ENSE. (2017). Encuesta Nacional De Salud. ESPAÑA 2017 (ENSE 2017). Recuperado de http:/ / www.mscbs.gob.es/ estadEstudios/estadisticas/encuestaNacional/encuestaNac2017/ENSE17_MOD3_REL.pdf.

Edwards, E. A., Lumsden, J., Rivas, C., Steed, L., Edwards, L. A., Thiyagarajan, A.,... Walton, R. T. (2016). Gamification for health promotion: Systematic review of behaviour change techniques in smartphone apps. BMJ open, 6, e012447. doi:10.1136/bmjopen-2016-012447.

Fiore, M. C., Jaén, C. R., Baker, T. B., Bailey, W., Benowitz, N., Curry, S.,... Leitzke, C. (2008). Treating tobacco use and dependence: 2008 update. Public Health service clinical practice guideline executive summary. Respiratory Care, 53, 1217-1222.

Goodchild, M., Nargis, N. y Tursan d'Espaignet, E. (2018). Global economic cost of smoking-attributable diseases. Tobacco Control, 27, 58-64. doi:10.1136/tobaccocontrol-2016-053305.

Gulati, G. y Hinds, B. (2018). Smoking cessation potential of smartphone-assisted behabioral therapy coupled to programmable carbon nanotuve membrane nicotine delivery device. Critical Reviews in Therapeutic Drug Carrier Systems, 35, 495-520. doi:10.1615/CritRevTherDrugCarrierSyst.2018020331.

Hartmann-boyce, J., Stead, L. F., Cahill, K. y Lancaster, T. (2013). Efficacy of interventions to combat tobacco addiction: Cochrane update of 2012 reviews. Addiction, 108, 1711-1721. doi:10.1111/add.12291.

Haskins, B. L., Lesperance, D., Gibbons, P. y Boudreaux, E. D. (2017). A systematic review of smartphone applications for smoking cessation. Translational Behavioral Medicine, 7, 292-299. doi:10.1007/s13142-017-0492-2.

Heather, N., Haffe, J. H., Peele, S. y Rho-, T. (2016). La adicción "no" es una enfermedad cerebral. Papeles del Psicólogo, 37, 118-125.

Heffner, J. L., Vilardaga, R., Mercer, L. D., Kientz, J. A. y Bricker, J. B. (2015). Feature-level analysis of a novel smartphone application for smoking cessation. The American Journal of Drug and Alcohol Abuse, 41, 68-73. doi:10.3 109/00952990.2014.977486.

Jiménez-Treviño, L., Velasco, Á., Rodríguez-Revuelta, J., Abad, I., De La Fuente-Tomas, L., González-Blanco, L.,... Sáiz, P. A. (2019). Factors associated with tobacco consumption in patients with depression. Adicciones, 31, 298-308. doi:10.20882/adicciones.1191.

Kazdin, A. E. (2015). Technology-based interventions and reducing the burdens of mental illness: Perspectives and comments on the special series. Cognitive and Behavioral Practice, 22, 359-366. doi:10.1016/j.cbpra.2015.04.004.

Kreps, G. L. y Neuhauser, L. (2010). Patient education and counseling new directions in eHealth communication: 
Opportunities and challenges. Patient Education and Counseling, 78, 329-336. doi:10.1016/j.pec.2010.01.013.

Leal-López, E., Sánchez-Queija, I. y Moreno, C. (2019). Trend in tobacco use among adolescents in Spain (20022018). Adicciones, 31, 289-297. doi:10.20882/adicciones.1111.

Liberati, A., Altman, D. G., Tetzlaff, J. y Al, E. (2009). The PRISMA statement for reporting systematic reviews and meta-analyses of studies that evaluate health care interventions: Explanation and elaboration. Journal Clinic Epidemiologic, 62, e1-34.

Lin, Y., Tudor-Sfetea, C., Siddiqui, S., Sherwani, Y., Ahmed, M. y Eisingerich, A. B. (2018). Effective behavioral changes through a digital mHealth app: Exploring the impact of hedonic well-being, psychological empowerment and inspiration. Journal of Medical Internet Research, 20. doi:10.2196/10024.

Luxton, D. D., Mccann, R. A., Bush, N. E., Mishkind, M. C. y Reger, G. M. (2011). mHealth for Mental Health: Integrating smartphone technology in behavioral healthcare. Professional Psychology: Research and Practice, 42, 505-512. doi:10.1037/a0024485.

Martínez-Vispo, C., Rodríguez-Cano, R., López-Durán, A., Senra, C., Fernández del Río, E. y Becoña, E. (2019). Cognitive-behavioral treatment with behavioral activation for smoking cessation: Randomized controlled trial. PLoS One, 14, e0214252. doi:10.1371/journal. pone.0214252.

Minami, H., Brinkman, H. R., Nahvi, S., Arnsten, J. H., Rivera-Mindt, M., Wetter, D.,... Brown, R. A. (2018). Rationale, design and pilot feasibility results of a smartphone-assisted, mindfulness-based intervention for smokers with mood disorders: Project mSMART MIND. Contemporary Clinical Trials, 66, 36-44. doi:10.1016/j. cct.2017.12.014.

Naughton, F. (2016). Delivering "Just-In-Time" smoking cessation support via mobile phones: Current knowledge and future directions. Nicotine E Tobacco Research, 19, 379-383. doi:10.1093/ntr/ntw143.

NICE. (2018). Stop smoking interventions and services. Recuperado de https://www.nice.org.uk/guidance/ ng92/resources/stop-smoking-interventions-and-services-pdf-1837751801029.

Nijhof, S. L., Bleijenberg, G., Uiterwaal, C. S. P. M., Kimpen, J. L. L. y Putte, E. M. Van De. (2012). Effectiveness of internet-based cognitive behavioural treatment for adolescents with chronic fatigue syndrome (FITNET): A randomised controlled trial. The Lancet, 379, 1412-1418. doi:10.1016/S0140-6736(12)60025-7.

Öberg, M., Jaakkola, M. S., Woodward, A., Peruga, A. y Prüss-Ustün, A. (2011). Worldwide burden of disease from exposure to second-hand smoke: A retrospective analysis of data from 192 countries. The Lancet, 377, 139146. doi:10.1016/S0140-6736(10)61388-8.
OMS (2019). La OMS destaca la enorme magnitud de la mortalidad por enfermedades pulmonares relacionadas con el tabaco. Recuperado de https://www.who.int/es/ news-room/detail/29-05-2019-who-highlights-huge-scale-of-tobacco-related-lung-disease-deaths.

Raich, A., Martínez-Sánchez, J., Marquilles, E., Rubio, L., Fu, M. y Fernández, E. (2015). Smoking cessation after 12 months with multi-component therapy. Adicciones, 27. doi:10.20882/adicciones.27.1.

Regmi, K., Kassim, N., Ahmad, N. y Tuah, N. A. (2017). Effectiveness of Mobile Apps for Smoking Cessation: A Review. Tobacco Prevention E Cessation, 3. doi:10.18332/ tpc/70088.

Stead, L. F., Koilpillai, P. y Lancaster, T. (2015). Additional behavioural support as an adjunct to pharmacotherapy for smoking cessation. Cochrane Database of Systematic Reviews, 10. doi:10.1002/14651858.CD009670.pub3.www. cochranelibrary.com.

Thomas, D., Abramson, M. J., Bonevski, B. y George, J. (2017). System change interventions for smoking cessation. Cochrane Database of Systematic Reviews, 2. doi:10.1002/14651858.CD010742.pub2.

Tudor-Sfetea, C., Rabee, R., Najim, M., Amin, N., Chadha, M., Jain, M.,... Eisingerich, A. B. (2018). Evaluation of two mobile health apps in the context of smoking cessation: Qualitative study of cognitive behavioral therapy (CBT) versus Non-CBT-based digital solutions. Journal of Medical Internet Research_Mhealth Uhealth, 6, e98. doi:10.2196/mhealth.9405.

UK Government. (2017). Cost of smoking to the NHS in England: 2015. Recuperado de https://www.gov.uk/ government/publications / cost-of-smoking-to-thenhs-in-england-2015/cost-of-smoking-to-the-nhs-inengland-2015.

Whittaker, R., McRobbie, H., Bullen, C., Rodgers, A. y Gu, Y. (2016). Mobile phone-based interventions for smoking cessation. The Cochrane Database of Systematic Reviews, 4, CD006611. doi:10.1002/14651858.CD006611.pub4. 